\title{
Valoración técnico económica de la tasa de Enfriamiento en soldadura vs la utilización de modelos MEF
}

\author{
Technical and economic assessment rate of \\ Cooling welding using models FEM \\ Alexander Pinilla Carvajal ${ }^{1}$ Laura Gallego Cossio $^{2}$ \\ Ludivia Hernandez Aros ${ }^{3}$ \\ ${ }^{I}$ Esp. Ingeniero Civil, Profesor de la Facultad de Ingeniería Civil, Universidad Cooperativa de Colombia, \\ Sede Ibagué. \\ alexander.pinillalcampusucc.edu.co \\ ${ }^{2}$ M BA. Contadora Pública, Profesora de la Facultad de Contaduría Pública, Universidad Cooperativa de \\ Colombia, Sede Ibagué. \\ laura.galgo@campusucc.edu.co \\ ${ }^{3}$ Esp. Contadora Pública, Profesora de la Facultad de Contaduría Pública, Universidad Cooperativa de \\ Colombia, Sede Ibagué. \\ ludivia.hernandez@campusucc.edu.co
}

\begin{abstract}
Resumen-El desarrollo de este artículo de investigación está orientado a establecer una comparación entre los resultados obtenidos vía experimental de la estimación de la tasa de enfriamiento en agua de probetas soldadas de geometría en $\mathbf{T}$ hechas de acero estructural ASTM HR A36, frente al análisis utilizando herramientas computacionales, particularmente el software de Modelamiento de elementos finitos (MEF) ANSYS, para realizar el ensayo experimental se utilizan termocuplas tipo $K$ las cuales captan valores de temperatura al enfriamiento que se obtienen por medio de tarjetas de adquisición de datos, el análisis del modelo MEF requirió del desarrollo de una malla mayor a 850.000 nodos, los resultados producto del análisis comparativo de las tasa de enfriamiento, presentan una diferencia entre métodos inferior a $3 \%$, lo cual permite estimar a la alternativa computacional como confiable al momento de establecer este tipo de análisis, adicionalmente se evalúan los costos asociados al desarrollo de ensayos experimentales vs la aplicación de modelos MEF, para ello se determinan los costos asociados a las etapas de experimentación, se establece que la tasa de recuperación, solo para el caso de ensayos de enfriamiento es de 15 meses, sin embargo la versatilidad de esta herramienta computacional, permite explorar otros usos.
\end{abstract}

Palabras clave- Análisis técnico económico, soldadura, enfriamiento.8.

Abstract- The development of this research paper is focused on a comparison between the results obtained experimentally for the estimation of the rate of cooling water welded geometry $T$ specimens made of structural steel ASTM HR A36 compared to analyzes using computational tools, particularly the software of the finite element modeling (FEM) ANSYS, the experimental test for type $\mathrm{K}$ thermocouples which capture the cooling temperature values obtained by the data acquisition cards are used, the analysis model required MEF the development of a more than 850,000 mesh nodes, the results of the comparative product analysis of the cooling rate, showing a difference between methods below $3 \%$, which allows the computer to estimate as a reliable alternative when setting this type of analysis additionally the costs associated with the development of experimental tests vs MEF implementing models for the costs associated with this stage of experimentation are determined, it is established that the rate of recovery, just to test cooling is evaluated 15 months, but the versatility of this computational tool, allows you to explore other uses.

Key Word - Technical and economic analysis, welding, cooling.

\section{INTRODUCCIÓN}

La utilización de modelos de elementos finitos (MEF), para la comprobación teórica de ensayos experimentales, es una práctica de uso común en ingeniería, esta a su vez ha venido cobrando más relevancia debido a la mayor capacidad de los equipos de cómputo lo cual provee mejor desempeño al momento de realizar el procesamiento [1]. La soldadura es un procedimiento ampliamente utilizado en el proceso de unión de materiales y posee múltiples aplicaciones industriales, existen algunas limitaciones que afectan a este, y en su 
mayoría se deben a las etapas de enfriamiento posteriores a la deposición de los cordones soldados, entre estos se tienen; defectos en los cordones de soldadura los cuales influyen en las propiedades mecánicas, y aparición de esfuerzos residuales y deformaciones de la microestructura de la soldadura, esto principalmente originado debido a un calentamiento local durante el proceso de soldadura [2].

Las tensiones residuales y las distorsiones son los principales problemas que se producen debido al efecto del calor en la soldadura. Las pruebas experimentales son una forma común de reducir al mínimo estos problemas, sin embargo esto conlleva un costo significativo de material y recursos operativos. Desde la perspectiva económica si se dispone de suficiente tiempo y los recursos apropiados es posible realizar la cantidad de ensayos que se requieran sin embargo en la mayor parte de las ocasiones este no es el caso, para ello una forma de disminuir este costo es utilizando herramientas computacionales MEF. Consecuentemente aumenta el costo computacional, existe una gran cantidad de software disponibles para este tipo de procesamiento entre otros están los programas con licencias General Public License o licencia pública general (GPL), con una amplia variedad de software de elementos finitos, con diferentes características, capacidades, campos de aplicación, modos y plataformas de trabajo, todos tienen en común el libre uso y acceso a los códigos fuente, que le posibilitan al usuario el mejoramiento y adaptación del programa a sus necesidades particulares, entre estos se encuentran, Calculix, CodeAster, EDF, Tochnog, FreeFem, FElt [3]. También hay una amplia selección de software comercial estos se encuentran restringidos en cuanto al código de procesamiento sin embargo se caracterizan por la fiabilidad de los resultados y la capacidad de cálculo, entre otros se destaca, Abaqus, Cosmos, Ansys, siendo este último el software que se utiliza en el estudio, teniendo en cuenta que una de las tareas que consume tiempo importante en la simulación de soldadura es la calibración del campo de temperatura para la cual se realiza un enfoque experimental contra datos obtenidos de las simulaciones [4].

Con el propósito de realizar una evaluación detallada de la utilización de herramientas computacionales MEF como alternativa a los ensayos experimentales desde la perspectiva del costo económico se tiene que según diversos autores (Sapag, 1993; Sapag y Sapag, 1995; Nicholson, 1997; Sandoval, 1998; Fontaine, 1998), los principales criterios e indicadores para estimar la recuperación de una inversión en software son los siguientes: Tasa Interna de Retorno (TIR), Índice de Valor Actual Neto (IVAN), y Período de Recuperación de la Inversión (PRI) [5,6,7].

La tasa interna de retorno o tasa interna de rentabilidad (TIR) de una inversión, expresa la "Rentabilidad Bruta" del mismo en términos relativos y por periodo; siendo una tasa de actualización o descuento que equipara el valor actual del flujo de egresos con el valor actual del flujo de ingresos. Como criterio de aceptación se tiene que si la tasa de rendimiento del proyecto expresada por la TIR supera la tasa de corte, se acepta la inversión; en caso contrario, se rechaza [8].

\section{MATERIALES Y MÉTODOS}

Se utilizaron probetas en forma de T las cuales utilizan como material base acero estructural ASTM HR A36 y como material de aporte electrodo E6013, el procedimiento empleado para la unión de las chapas es la soldadura manual con electrodo revestido (SMAW) es la más ampliamente usada en el montaje estructural.

\section{A. Geometría y características de la probeta}

Para el desarrollo experimental se utilizó una chapa de acero al carbono ASTM HR A36 comercial, de espesor $8 \mathrm{~mm}$ cuya composición química corresponde a la presentada en la Tabla 1 .

Tabla 1: Composición química del acero ASTM HR A36 empleado (Fuente: Compañía Nacional de Aceros, código material 15833)

\begin{tabular}{ccccccccc}
\hline $\mathbf{F e}$ & $\mathbf{C}$ & $\mathbf{S i}$ & $\mathbf{M n}$ & $\mathbf{S}$ & $\mathbf{P}$ & $\mathbf{N i}$ & $\mathbf{C r}$ & $\mathbf{N}$ \\
\hline 95.425 & 0.16 & 0.17 & 0.78 & 0.09 & 0.010 & 0.025 & 0.04 & 0.065 \\
\hline
\end{tabular}

El proceso de soldadura se realizó empleando un equipo de soldadura marca MAXWELD 160 D.C. Se llevaron a cabo 2 depósitos de filete, por ambos lados con bisel cuadrado, aplicando catetos de $4 \mathrm{~mm}$. Las características eléctricas del proceso SMAW son mostradas en la Tabla 2, para cada tamaño de cateto de soldadura.

Tabla 2: Especificación de los procedimientos de soldadura utilizados

\begin{tabular}{cccc}
$\begin{array}{c}\text { Cateto } \\
\text { del } \\
\text { cordón } \\
\text { empleado }\end{array}$ & $\begin{array}{c}\text { Diámetro } \\
\text { del } \\
\text { electrodo } \\
\text { E-6013 }\end{array}$ & $\begin{array}{c}\text { Características } \\
\text { eléctricas }\end{array}$ & $\begin{array}{c}\text { Velocidad } \\
\text { de } \\
\text { Avance }\end{array}$ \\
\hline $4 \mathrm{~mm}$ & $1 / 8 ”$ & $\begin{array}{c}72 \text { voltios, } \\
94 \mathrm{~A} \mathrm{CD}\end{array}$ & $\begin{array}{c}22 \\
\mathrm{~cm} / \mathrm{min}\end{array}$ \\
& & &
\end{tabular}

La geometría y dimensiones de la probeta en $\mathrm{T}$ se presentan en la Figura 1.

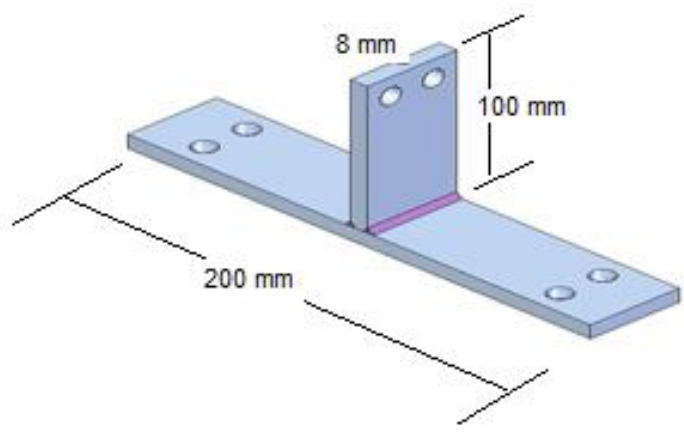


Figura 1: Geometría de la probeta soldada en T.

B. Montaje experimental para el ensayo de enfriamiento

La medición de la temperatura de la probeta durante su enfriamiento en agua se realizó utilizando dos termocuplas tipo K, ubicadas lateralmente. Las termocuplas se conectan a una tarjeta de adquisición de datos NI-9211, montada sobre el chasis NI-CDAQ-9172 y luego al PC como aparece en el diagrama presentado en la Figura 2. Para la visualización de los resultados se emplea el software LabView Signal Express 2013.

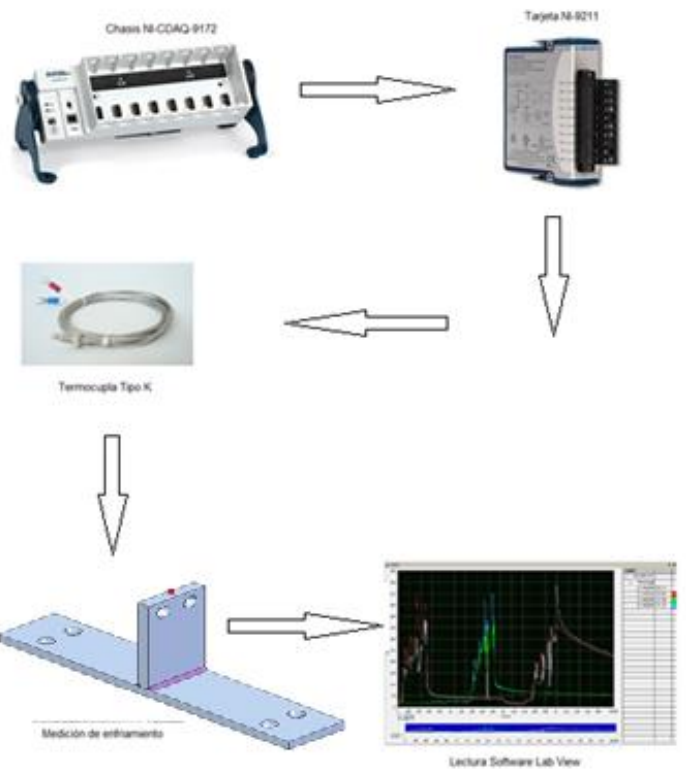

Figura 2: Montaje experimental para la medición de la temperatura.

En el diagrama de bloques que aparece en la Figura 3 se define el procedimiento de modelamiento empleado. Se emplea el Software ANSYS® V14.5 para la determinación de la curva de enfriamiento en el medio agua.

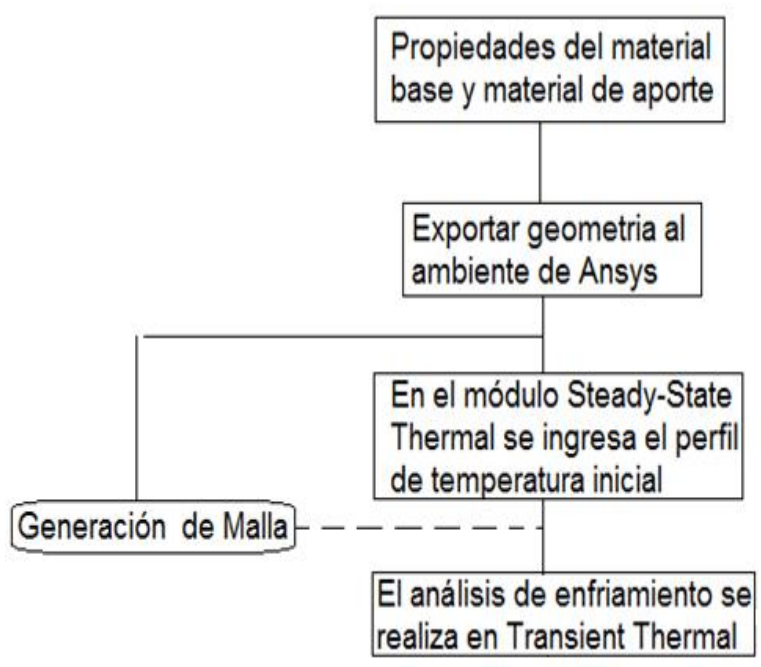

Figura 3. Diagrama de bloques para el procedimiento de modelamiento empleado

\section{EXPERIMENTACIÓN Y MODELAMIENTO}

En la Figura 4 se muestra una imagen del ambiente grafico aplicado en el software de elementos finitos ANSYS 14.5

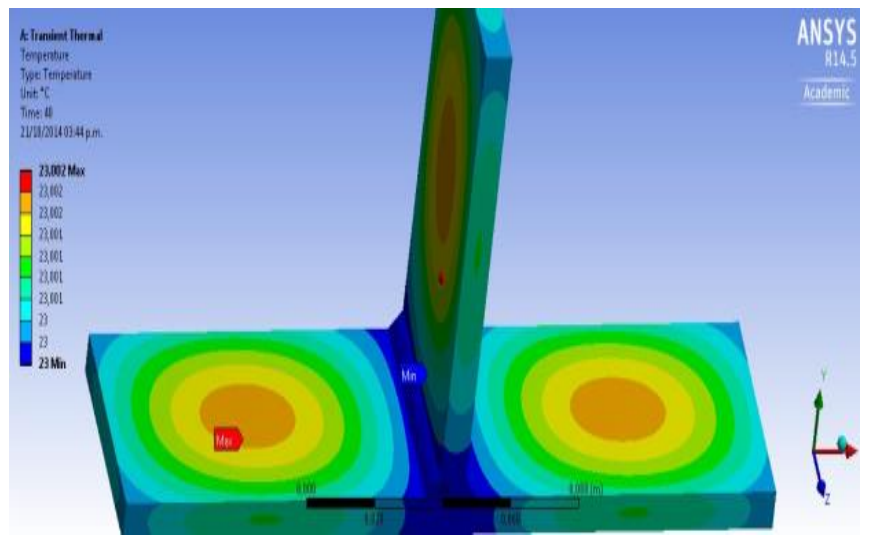

Figura 4. Probeta modelada en ANSYS 14.5

El método de análisis MEF se ha desarrollado en paralelo con los ensayos experimentales. Se obtuvo el comportamiento del campo de temperatura al momento del enfriamiento en agua de manera experimental, este comportamiento se ilustra en la Figura 5, a su vez se corrobora el ensayo aplicado utilizando el software de MEF ANSYS para una malla de 853.467 nodos, en la figura 6 se ilustra la curva obtenida producto del modelamiento. 


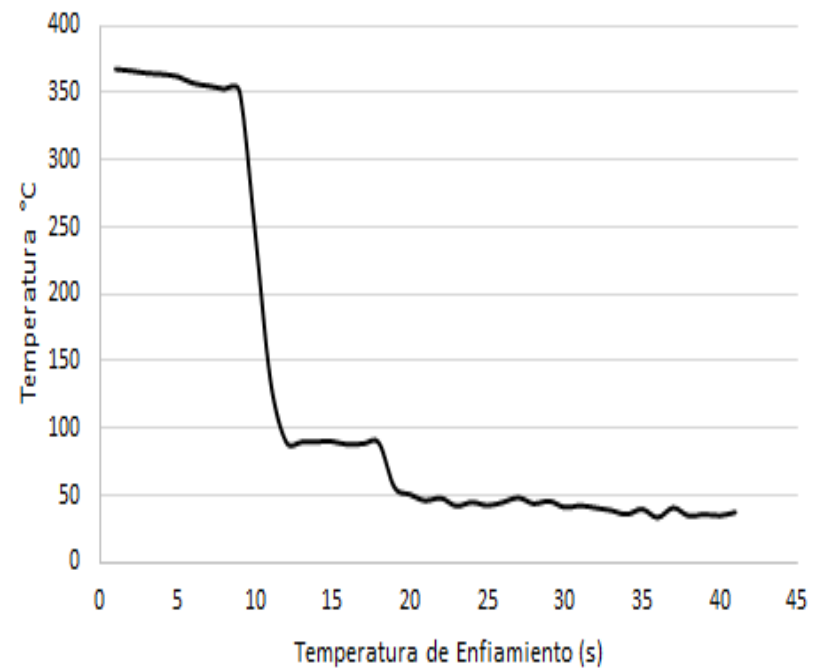

Figura 5. Curva de enfriamiento experimental con medio agua y cateto de soldadura de $4 \mathrm{~mm}$.

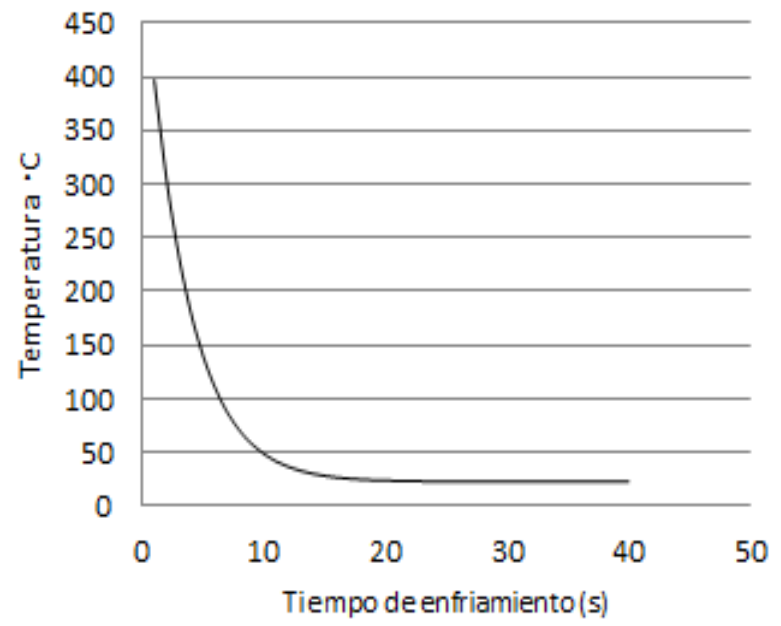

Figura 6. Curva de enfriamiento teórica con medio agua y cateto de soldadura de $4 \mathrm{~mm}$.

\section{RESULTADOS Y DISCUSIÓN}

El objetivo principal de este trabajo es investigar la forma analítica y los enfoques numéricos que se pueden utilizar de forma complementaria, para estimar la razón de enfriamiento en agua de una unión soldada en T. Sobre la utilización de técnicas MEF en la actualidad es posible afirmar que tradicionalmente la concepción de futuro es un escenario al cual inevitablemente se va a llegar por eso es importante conocer qué futuro es el que se vendrá a fin de tomar las acciones respectivas [9].

Se observa un comportamiento similar entre las curvas de enfriamiento producto de las simulaciones y las registradas mediante los ensayos experimentales. En la Tabla 3 se identifica esta correspondencia
Tabla 3. Tasa de enfriamiento $\left({ }^{\circ} \mathrm{C} / \mathrm{s}\right)$.

\begin{tabular}{lcc}
\hline \multirow{2}{*}{$\begin{array}{c}\text { Medio de } \\
\text { enfriamiento }\end{array}$} & \multicolumn{2}{c}{ Modelo } \\
\cline { 2 - 3 } & Experimental & Teórico \\
\hline Agua & 9.3678 & 10.4238 \\
\hline
\end{tabular}

De manera complementaria se estima el costo que infiere el uso de herramientas computacionales respecto a la utilización de ensayos experimentales.

\section{A. Análisis Económico}

El desarrollo de este análisis requiere el establecimiento de los costos producto de la adquisición del software MEF y los costos respectivos debido al ensayo experimental de enfriamiento, estos costos deben incluir las repeticiones y el tiempo incluido para el desarrollo de cada análisis. En la tabla 4 se muestra el costo asociado a estas inversiones.

Tabla 4. Costos de los ensayos

\section{Tipo de ensayo}

\begin{tabular}{|c|c|c|}
\hline \multirow{3}{*}{ Insumo } & & \\
\hline & \multirow{2}{*}{$\begin{array}{c}\text { Experimental } \\
\operatorname{pesos}(\$)\end{array}$} & \multirow{2}{*}{$\begin{array}{c}\text { Teórico } \\
\text { Pesos (\$) }\end{array}$} \\
\hline & & \\
\hline $\begin{array}{l}\text { Chapas de } \\
\text { acero }\end{array}$ & 50.000 & $\mathrm{X}$ \\
\hline & & $X$ \\
\hline Electrodo & 5.000 & \\
\hline Soldador & 25.000 & $\mathrm{X}$ \\
\hline $\begin{array}{l}\text { Software } \\
\text { MEF }\end{array}$ & $\mathrm{X}$ & 6.000 .000 \\
\hline $\begin{array}{l}\text { Tiempo de } \\
\text { procesamiento }\end{array}$ & $\mathrm{X}$ & 12.000 \\
\hline TOTAL & 80.000 & 6.012 .000 \\
\hline
\end{tabular}

Al evaluar el costo asociado a ambos tipos de ensayos se obtiene que la recuperación de la inversión en una herramienta computacional respecto al uso de ensayos experimentales con una periodicidad mensual de 5 ensayos, se obtiene como se muestra en la tabla 5. 
Tabla 5. Valuación de las inversiones

\begin{tabular}{ccc}
\hline & $\begin{array}{c}\text { Ensayo } \\
\text { experiment } \\
\text { al }\end{array}$ & $\begin{array}{c}\text { Herramienta } \\
\text { MEF }\end{array}$ \\
\hline PRI & 1 mes & 15 meses \\
\hline Costo Total & $\$ 400.000$ & $\$ 6.012 .000$ \\
\hline
\end{tabular}

Se logra evidenciar que la inversión en herramienta computacional a largo plazo es una mejor opción que el uso continuado de ensayos experimentales debido a que posterior a esta inversión y el plazo de recuperación, se reducen ostensiblemente los costos asociados al desarrollo de ensayos experimentales, en este caso particular el ensayo de enfriamiento en soldaduras.

\section{CONCLUSIONES}

Se logra determinar experimentalmente la razón de enfriamiento para probetas en $\mathrm{T}$, enfriadas en agua utilizando métodos experimentales, se observa que la tasa de enfriamiento es de $9.3678{ }^{\circ} \mathrm{C} / \mathrm{s}$, la comparación con los modelos computacionales, establecen una diferencia menor al $3 \%$ lo cual es bastante acertado y puede ser aceptado como técnica alternativa a los ensayos experimentales.

La elección de la técnica MEF como alternativa al uso de ensayos experimentales, depende en gran medida de la capacidad de adquisición de tecnología, sin embargo se observa que el índice de recuperación de la inversión es bastante bueno y menor a 15 meses, permitiéndose obtener una herramienta versátil con la cual explorar otras alternativas de ensayos.

Debido a los cambios en los ciclos térmicos por el calentamiento y enfriamiento (no uniformes) en los materiales al ser soldados, se presentan tensiones residuales y deformaciones plásticas en las soldaduras. Estas tensiones residuales pueden ser afectadas por la rapidez del enfriamiento, es así como diversos medios de enfriamiento provocan diferentes valores en la tasa de enfriamiento, lo cual en dependencia de la intensidad o rapidez del mismo pueden llegar a afectar la integridad de los elementos soldados, problemas de fatiga reduciendo la vida útil de servicio, roturas frágiles y corrosiones.

\section{AGRADECIMIENTOS}

A la oficina de investigaciones de la Universidad Cooperativa de Colombia Sede Ibagué, por su valioso aporte al desarrollo de esta investigación

\section{REFERENCIAS}

[1]. M. Attarha,, I. Sattari-far, "Study on welding temperature distribution in thin welded plates through experimental measurements and finite element simulation". Journal of Materials Processing Technology. $\mathrm{N}^{\circ} 211:$ 688-694. 2011

[2]. S. Murugan, P.V. Kumar, B. Raj, Bose, "Temperature distribution during multi-pass welding of plates". Int. J. Press. Vessel N 75: 891-905. 1988.

[3]. C. Galeano, J. Mantilla, C. Duque, M. Mejía, "Herramientas de software con licencia pública general para el modelado por elementos finitos". DYNA, Vol. 74, núm. 153. 2007.

[4]. W. Perret, C. Schwenk, C. Rethmeier, "Comparison of analytical and numerical welding temperature field calculation". Computational Materials Science, $\mathrm{N}^{\circ}$ 47: 1005-1015. 2010.

[5]. O. Araque, L. Gallego," Análisis técnico económico de la implementación de mejoras en el procesamiento de carbonato de calcio," Scientia et Technica, Año XIX, No 1, pp. 42-48, Mar. 2014.

[6]. A. Lobos, G. Soto, R. Zenteno, N. Prizant, “'Análisis de eficiencia y rentabilidad económica en dos lecherías de la región del maule", Chile. Agricultura Técnica. Vol.61, n.3, pp. 367-378. 2001.

[7]. G. Leon, "Análisis comparativo de los métodos tradicionales de valoració aplicado a la simulación de un proyecto de inversión", Dimensión Empresarial, Vol. 10 No. 1, Enero - Junio. págs. 16-21. 2012.

[8]. J. Montllor, "Generación de renta, selección de inversiones y objetivos empresariales", Revista Española de Financiación y Contabilidad, Vol. XVII, n. 53, 1987, pp. 391-414. 1987.

[9]. A. Chung, "Cientificidad de la prospectiva: discusiones sobre su objeto de estudio", Revista de la facultad de Ingeniería Industrial, Vol. 15, n. 2: 116119. 2012. 